\title{
Analysis of Channel Coding Performance in OFDMTechnique for Underwater Acoustic Communication System
}

\author{
Machmud Roby Alhamidi' ${ }^{1}$ Gede Puja Astawa², Tri Budi Santoso3 \\ 1Student of D4 Study Program of Telecommunication Engineering \\ PoliteknikElektronikaNegeri Surabaya (PENS) \\ 2,3Lecturer of Study Program of Telecommunication Engineering \\ PoliteknikElektronikaNegeri Surabaya (PENS) \\ Jl.Raya ITS, Kampus PENS, Sukolilo \\ E-mail: machmudrobyalhamidi@gmail.com, \\ puja@eepis-its.edu, tribudi@eepis-its.edu
}

\begin{abstract}
One way to increase the performance of Orthogonal Frequency Division Multiplexing System (OFDM) system is by adding a channel coding (error correction code) in order to detect and correct errors that occur when sending data.At communication of acoustic underwater channel coding is required because of the characteristics of the channel bottom water is much different compared with the air channel and errors are likely to occur.In this research it was made simulation of acoustic underwater communication system with OFDM applied channel codingin which using Hamming code $(7,4)$ and Hamming code $(15,11)$ that is able to correct one error and detect two errors then $\mathrm{BCH}$ code capable to correct two errors for BCH $(15,7)$ and correct 9 errors forBCH $(127,64)$ and Reed Solomon code able to correct two errors for RS $(15,11)$ and correct 8 errors for RS $(31,15)$. Results of the study confirm the better performance when system usesOFDM with BCH Code (127.64) than other codes that are used, starting from 1 decibel $(\mathrm{dB})$ to $3 \mathrm{~dB}$ for the performance of BER as $10^{-3}$ on Additive Gaussian White Noise (AWGN) channel while at the multipath channel, the performance of Bit Error Rate (BER) got better result on $1 \mathrm{~dB}$ up to $8 \mathrm{~dB}$ for BER performance as $10^{-3}$.
\end{abstract}

Keyword: Underwater, Orthogonal Frequency Division Multiplexing (OFDM), channel coding.

\section{INTRODUCTION}

Alkydiz [1] in his paper presents a state of the art underwater sensor networks, and gives an overview about challenges of underwater acoustic channel in the monitoringsystem the environmental conditions of the ocean bottom. Itis also given directives for researchers in building a underwater 
sensor networks systems to produce a reliable underwater communication system in application of environment monitoring and exploitation of undersea natural resources. Stojanovic and Citre [2] in theirpapers have given direction for the research of underwater acoustic communication. Manjula and Sunilkumar [3] in theirpapers have given a description to create an underwater applications which able to work well, it takes an efficient communication protocol on underwater equipment based on wireless technology, due to the high attenuation and scattering processes that affect radio waves. In this paper it is also discussed about the underwater communication system usingacoustic signals. JordiRibas and Daniel Sura [4] in their paper reporting discusses how video transmission on the underwater channel where to improve the transmission performance using not only OFDM but also channel coding such as BCH code with various code structure which can fix error that occur when sending data through the underwater channel. The appropriate channel coding can increase performance and efficiency ofsystem, particularly on the underwater channel withhigh damping, limited bandwidth and also there is a Doppler effect. In addition, the presence of noise that occurs because of a limitation hardware and high damping [6].

\section{Modeling of Transmission System of Underwater Acoustic Channel}

Figure 1 showsblock diagram block of OFDMsystem using channel coding (corrections error codes) on underwater multipathchannels. Systems are divided into three main systems namely systems on transmitter OFDM, underwater channel and system in receiver OFDM [6].

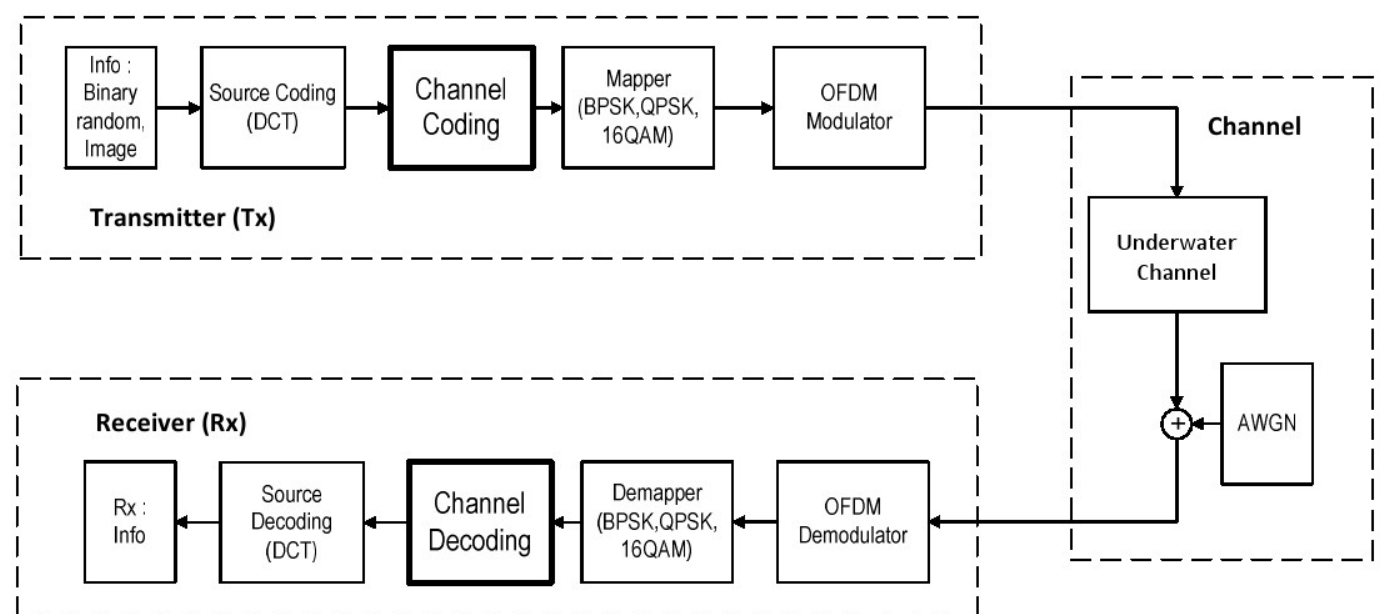

Figure 1.Block diagram simulation system

\subsection{Transmitter System OFDM}

In the transmitter system, there is a generation of information signal such as binary random with number of bit 616.000 bit and image gray scale at size $273 \times 184$ pixel. 


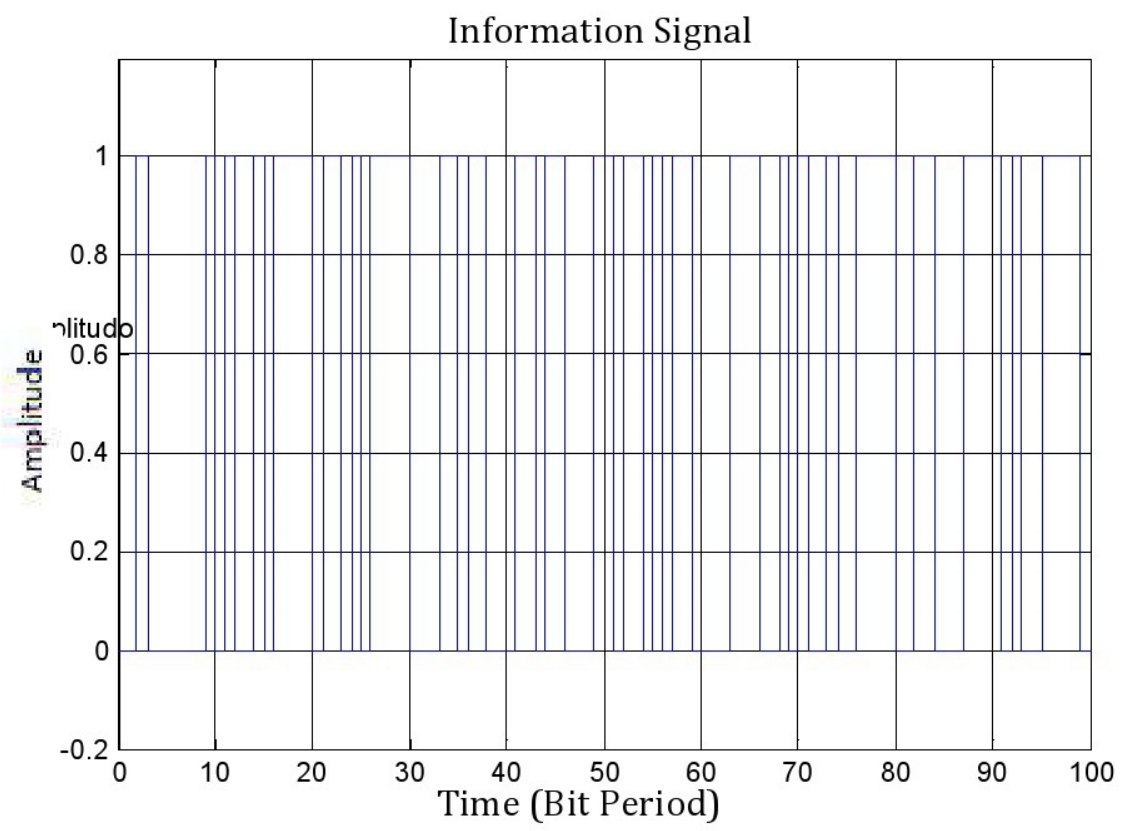

(a)

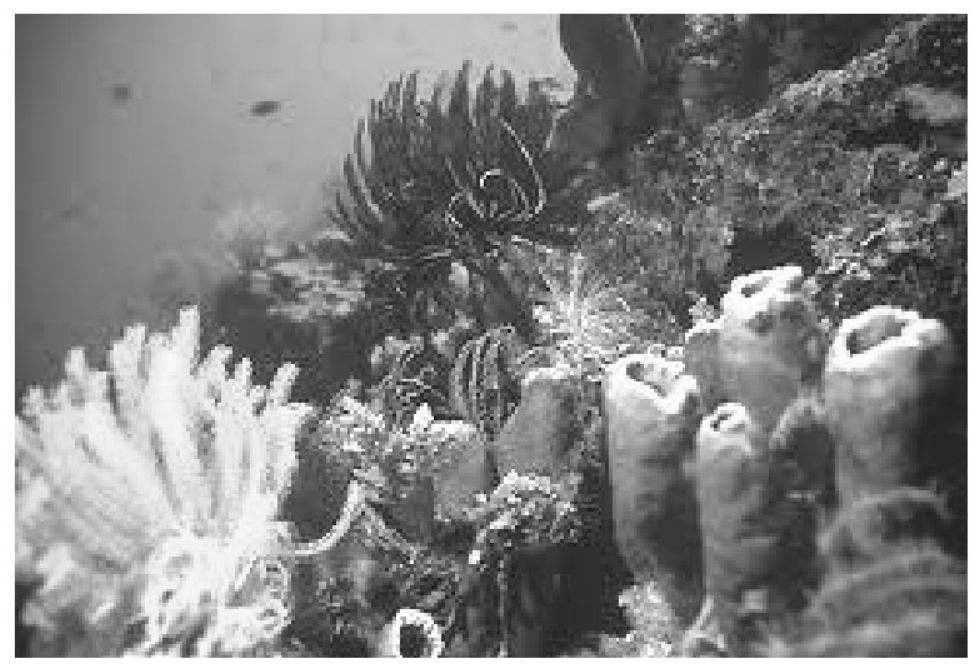

(b)

Figure2.Information signal (a) Binary random (b) Image grey scale

For image data it has been conducted process source coding process using Discrete Cosine Transform (DCT) technique with dividing image of grey scale into block 8x8 where each block present spatial domain(distance) into frequency domain(frequency of emergence grey scale) by applying equation (1) [8].

$$
C(u, v)=a(u) a(v) \sum_{x=0}^{M-1} \sum_{y=0}^{N-1} f(x, y) \cos \left[\frac{\pi(2 x+1) u}{2 M}\right] \cos \left[\frac{\pi(2 y+1) u}{2 N}\right]
$$

where $\mathrm{C}(\mathrm{u}, \mathrm{v})$ is the coordinatespoint of the matrix which has undergoneDCT 
transformation two-dimensional. $\mathrm{M}$ and $\mathrm{N}$ are number of columns and rows, $\mathrm{a}(\mathrm{u})$ and $(\mathrm{v})$ is result repositorywith value determined from coefficient value of $u$ and $v, f(x, y)$ is pixel value of matrix at point $(x, y)$, valued at $180^{\circ}$. The next process is the formation of code-word with channel coding, for Hamming code for (n, k) formation of a code-word can be obtained from equation (2) [7].

$$
\text { C=d.G }
$$

where $G$ is generator matrix for Harming code $(n, k)$ and $d$ is bit input encoded.

Generator matrix for Harming code is able to be generated by combining between bits of identity matrix and bits of parity matrix.The evocation of matrix gen erator is shown by equation (3) [7].

$$
G=\left[P_{k x(n-k)}^{T} \quad \vdots \quad I_{k}\right]
$$

The process of forming a codewordfor BCH code (n,k). Bits from source data entering to encoderare encoded with applying generator matrix. The general form of generator matrix of $\mathrm{BCH}$ code is shown in equation (4) [7].

$$
G=\left[P_{k x(n-k)} \quad \vdots \quad I_{k}\right]
$$

The process of codeword formation with Reed Solomon code (n,k,m) started by taking $\mathrm{k}$ bit data as much as *k bit and then changed into symbol where each $\mathrm{m}$ bit represent one symbol.Furthermore, that symbols crossed with generator polynomial which formulated in equation $(5)\left(\left(x+a^{t}\right)\right.$ is minimum polynomial of $\mathrm{a}^{\mathrm{t}}$ )[7]

$$
\begin{aligned}
& g(X)=g_{0}+g_{1} X+g_{2} X^{2}+\cdots+g_{2 t-1} X^{2 t-1}+X^{2 t}=\left(X+\alpha^{1}\right)(X+ \\
& \left.\alpha^{2}\right) \ldots\left(X+\alpha^{2 t}\right)
\end{aligned}
$$

where $\alpha$ is element primitiveof $\mathrm{GF}\left(2^{\mathrm{m}}\right)$ and $\alpha^{1}, \alpha^{2}, \ldots, \alpha^{2 t}$ is element of similarfield.

Process on OFDM started from serial to parallel process where changethe data originally serial to be parallel as number ofsize of Fast Fourier Transform (FFT) (in this case the size of FFT is 256), in this process performed the process of pilot symbol insertion that serves as a guard between one carrier and others (on OFDM useful for removing ICI)

The process of forming a symbol in OFDM using Inverse Fast Fourier Transform (IFFT) that change one frequency become orthogonal against other frequency. A process at IFFT can be shown by equations (6)[9][10].

$$
x(l)=\frac{1}{K} \sum_{k=0}^{K-1} X(k) e^{j 2 \pi k l / K}
$$


wherex $(l)$ is signal output IFFT, then $\mathrm{X}(\mathrm{k})$ is signal is sent at time $\mathrm{k}$ and $\mathrm{K}$ is number of subcarrier.

After forming OFDM symbol, next process is addition of cyclic prefix as channel guard so ISI never happened. After that, data are transformed again into serial so it is able to be sent through channel with parallel to serial converter.

\subsection{Underwater MultipathChannel}

Data that has been processed in the sender subsequently sent through the underwater channel. The underwater channel is simulated as a channel with multipath fading and distribution of Rayleigh. Rayleigh fading is obtained by rapidly amplitude signal fluctuations in a certain period of time caused by receipt of two or more similar signals by the receiver due to the plural. Additionally, on underwater multipath channel there is also anaddition of AWGN noise where the AWGN is a noise with additive character, which has function of a constant power spectral density for all frequencies (white noise) and Gaussian probability.

\subsection{Receiver System OFDM}

The first process conducted in demodulation OFDM is process of process serial to parallel converter that change serial datato parallel dataand for next step is vanishing cyclic prefix. Process of unravelling symbol at OFDM using Fast Fourier Transform (FFT) to elicit signals in the frequency domain, process in FFT can be shown by equation (7)[9].

$$
X(k)=\sum_{l=0}^{K-1} x(l) e^{-j 2 \pi k l / K}
$$

where $\mathrm{x}(l)$ is signal at time $l$, then $\mathrm{K}$ is number of subcarrier(value of subcarrier assumed 256),and $\mathrm{k}$ is frequency index of $\mathrm{K}$ amount of frequency and $\mathrm{l}$ is time index and yield $\mathrm{X}(\mathrm{k})$ as a value of spectrum for frequency $\mathrm{k}$.

Decodingprocess is a data recoding process that is coded before by using similar code and similar structure when coding process. For recoding processcodewordwhich is accepted $(\mathrm{r})$ with Hamming code $(\mathrm{n}, \mathrm{k})$ must be recognized syndrome. Where syndromeis able to be attained from equation (8)[7].

$$
S=r \cdot H^{T}
$$

where $\mathrm{H}^{\mathrm{T}}$ is transpose of parity checkmatrix. And that matrix can be obtained from equation (9)[7].

$$
H=\left[I_{k} \quad \vdots \quad P_{k x(n-k)}^{T}\right]
$$


From equation [8] obtainedwhen all element $\mathrm{s}$ is 0 , then codewordhas been accepted correctly. But if s contain bit 1, so bit error adjusted by parity check analysis which one has error.

For recoding codeword which has been received with $\mathrm{BCH}$ code $(\mathrm{n}, \mathrm{k})$ and $n=15$ conducted syndrome calculation with equation (10)[7].

$$
\begin{aligned}
& S_{1}=r_{0}+r_{1} a^{1}+r_{2} a^{2}+\cdots+r_{14} a^{14} \\
& S_{3}=r_{0}+r_{1}\left(a^{3}\right)^{1}+r_{2}\left(a^{3}\right)^{2}+\cdots+r_{14}\left(a^{3}\right)^{14}
\end{aligned}
$$

If the first syndrome $\left(\mathrm{S}_{1}\right)$ and the third syndrome $\left(\mathrm{S}_{3}\right)$ are zero, decoder doesn't do correction. But if it is not zero, next process is arrangingerror locatorpolynomial such as equation (11)[7]:

$$
\begin{gathered}
\sigma(x)=1+\sigma_{1} x+\sigma_{2} x^{2} \\
\text { where, } \sigma_{1}=S_{1} \text { and } \sigma_{2}=S_{1}^{2}+\frac{S_{3}}{s_{1}}
\end{gathered}
$$

It is found error position by invert value of roots of equation. After that the error bits are repaired by changing error bits (if previous bit is 0 then become bit 1 and bit 1 become bit 0 ).

For recoding codewordreceived with Reed Solomoncode $(\mathrm{n}, \mathrm{k}, \mathrm{m})$ and $\mathrm{n}=15$ conducted syndrome calculation with equation(12)[7].

$$
\begin{aligned}
& S_{1}=r_{0}+r_{1} a^{1}+r_{2} a^{2}+\cdots+r_{14} a^{14} \\
& S_{2}=r_{0}+r_{1}\left(a^{2}\right)^{1}+r_{2}\left(a^{2}\right)^{2}+\cdots+r_{14}\left(a^{2}\right)^{14} \\
& S_{3}=r_{0}+r_{1}\left(a^{3}\right)^{1}+r_{2}\left(a^{3}\right)^{2}+\cdots+r_{14}\left(a^{3}\right)^{14} \\
& S_{4}=r_{0}+r_{1}\left(a^{4}\right)^{1}+r_{2}\left(a^{4}\right)^{2}+\cdots+r_{14}\left(a^{4}\right)^{14}
\end{aligned}
$$

Next step is testing all syndromes, and if all valued zero so it means that no error and parity bits throw out then data bits will be kept at array. But if not then the determinant matrix must be counted P[7]:

$$
P=\left[\begin{array}{ll}
S_{1} & S_{2} \\
S_{2} & S_{3}
\end{array}\right]
$$

When determinant matrix $\mathrm{P}$ is zero, error polynomial $\mathrm{e}(\mathrm{x})$ achieved with equation (14)[7]:

$$
e(x)=e_{i 1} x^{\log _{\alpha} \sigma}
$$

where error locator $\sigma=s 2 / s 1$ dan error magnitude $\mathrm{e}_{\mathrm{i} 1}=\mathrm{S}_{1} / \sigma$ when determinant matrixPis not zero, error polynomial $\mathrm{e}(\mathrm{x})$ obtained with equation (15)[7]:

$$
e(x)=e_{i 1} x^{\log _{\alpha} \beta_{1}^{-1}}+e_{i 2} x^{\log _{\alpha} \beta_{2}^{-1}}
$$


where $e_{i 1}$ and $e_{i 2}$ is error magnitude, $\beta_{1}^{-1}$ and $\beta_{2}{ }^{-2}$ is invertfromroots of equation $\sigma(x)=1+\sigma_{1} x+\sigma_{2} x^{2}$.

For getting the real code $c(x)$, sum the receipt code $r(x)$ witherror polynomial $\mathrm{e}(\mathrm{x})$ as written in equation (16)[7]:

$$
c(x)=r(x)+e(x)
$$

After having binary data, the first process for reconstructing is converting that binary datainto decimal form. Then conducted dequantization process, where quantization factor used must be similar with the factor used before data sent. After dequantization, this data will be again transformed be image data using DCT invert.

\section{RESULT}

This section compares the performance of BER of Hamming codes, BCH codes and ReedSolomon code with system OFDM at underwater channel. For Hamming code, the code structure used is Hamming $(7,4)$ and Hamming (15.11) and then for BCH code, the code structure used is $\mathrm{BCH}$ (15.7) and BCH $(127,64)$ and Reed Solomon code, the code structure used is RS $(15.11)$ and RS $(31,15)$.

Figure 3a shows the comparison of the performance of Channel Coding using modulation QPSK for OFDM system in AWGN channel. When Eb/N0 is worth $6 \mathrm{~dB}$, the BER performance for Hamming code (7.4) is $4.6 \times 10^{-3}$ which means that when sending 616.000 bits of data so there are 2.832 bits of data error then for Hamming codes (15.11) produces the performance of BER $9.7 \times 10^{-3}$ which means than when sending 616.000 bits of data so there are 5,768 bits of data error. For BCH code (15.7) produces the performance of BER of $10^{-3}$ which means that when sending 616.000 bits of data so there are 665 bits of data error on the recipient process, and then to $\mathrm{BCH}$ code (127.64) produces the performance of BER $8 \times 10^{-6}$ which means that when sending 616.000 bits of data so there are 5 bits of data error.

For code RS $(15.11)$ and code RS $(31,15)$ each has a performance of BER $1,1 \times 10^{-2}$ and $3 \times 10^{-4}$ where when there are 616.000 bits ofdata sent, there are 6.960 data bits and 198 bits of data are error. In Figure 3b is indicated performance comparison of channel coding with modulation QPSK at OFDM system the underwater channel. To get the performance of BER of $10^{-3}$ by using the $\mathrm{BCH}$ Code (127.64) need to $\mathrm{Eb} / \mathrm{NO}$ at rate $10.5 \mathrm{~dB}$ then to $\mathrm{BCH}$ codes (15.7) need to $\mathrm{Eb} / \mathrm{N} 0$ at rate $13.5 \mathrm{~dB}$. Hamming code for (15.11) with $\mathrm{Eb} / \mathrm{N} 018.5 \mathrm{~dB}$ can get performance of BER of $10^{-3}$, then for Hamming code (7.4) need to Eb/N0 as $17 \mathrm{~dB}$ and for RS code $(31,15)$ need to Eb/N0 $11.5 \mathrm{~dB}$ to get performance of BER of $10^{-3}$ while for RScode (15.11) need to Eb/N0 as $17.5 \mathrm{~dB}$. 
Graphics Performance BER Channel Coding OFDM QPSK in AWGN Channel

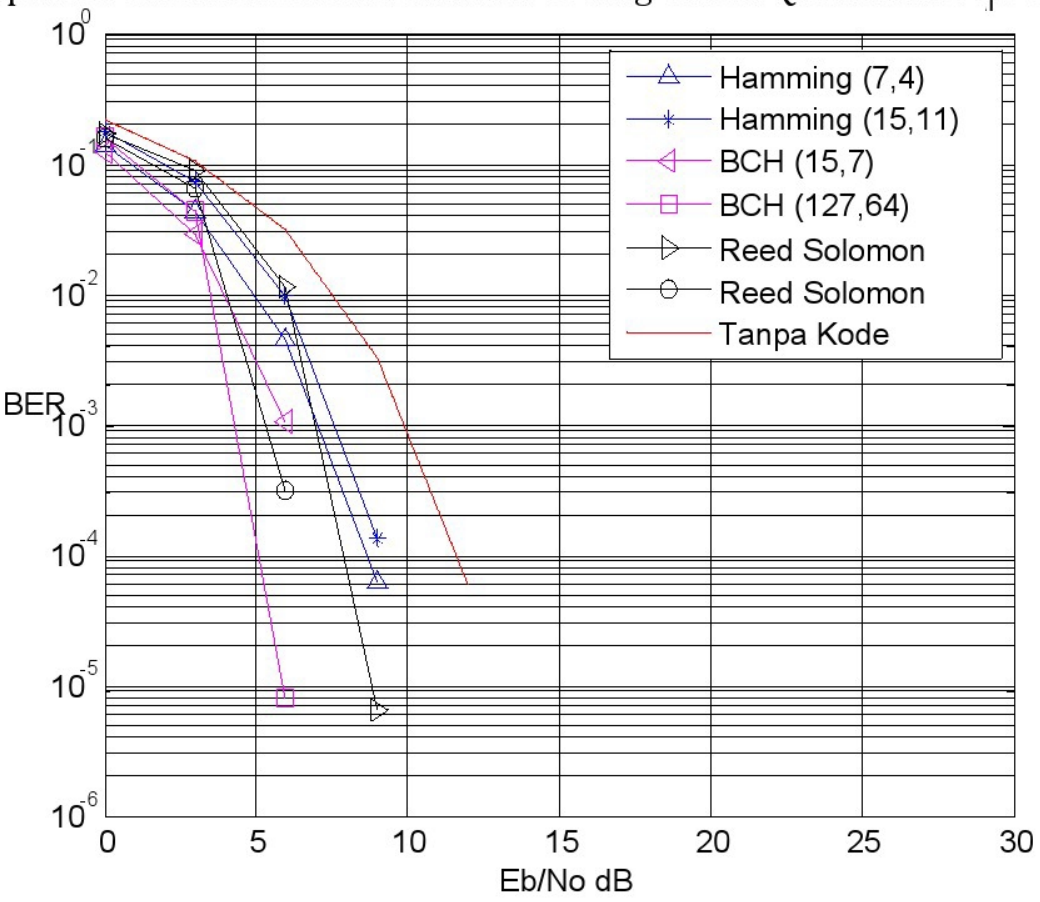

(a)

Graphics Performance BER Channel Coding OFDM QPSK in Multipatth88 Tap

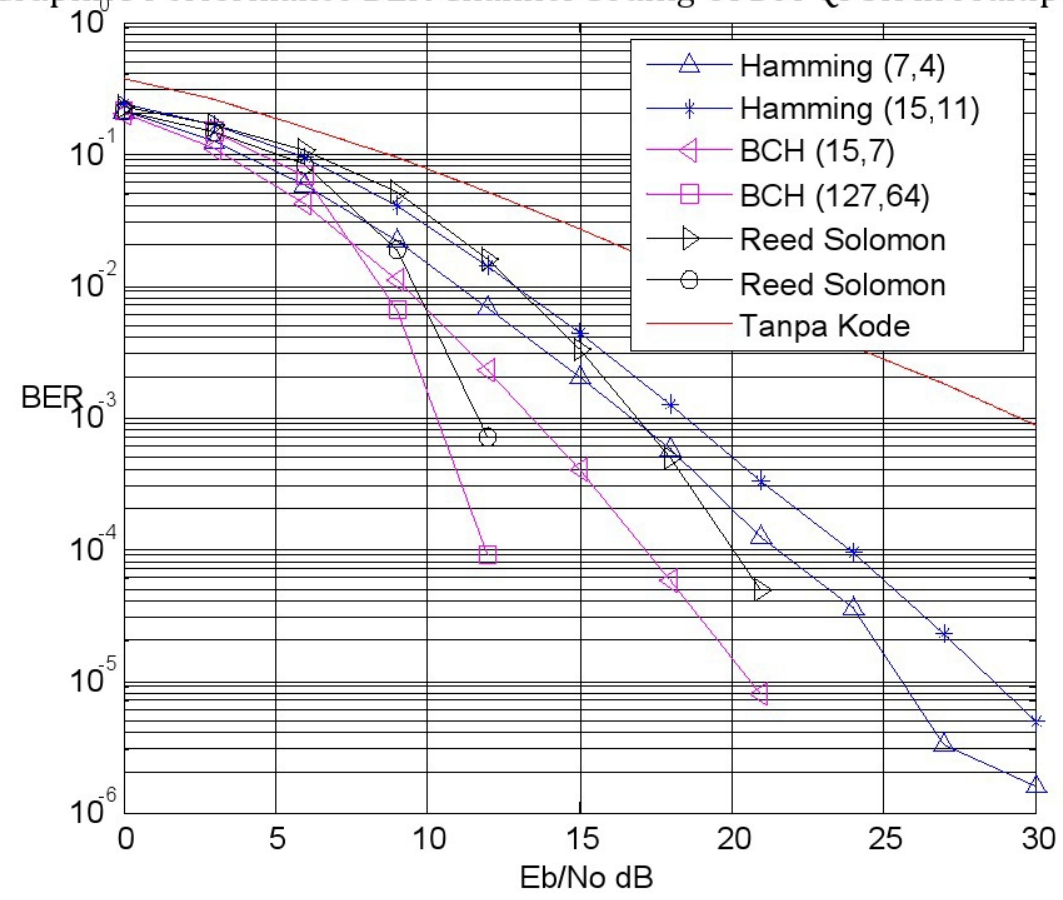

(b)

Figure3.Graph of performance comparison of BER channel codingOFDM;

(a) AWGN channel,(b) underwater channel 
In Figure 4 the results are obtained when the Eb/N0 as $9 \mathrm{~dB}$ using the Hamming code $(7,4)$ acquired PSNR performance as 9.65 . Then, when using Hamming code (15.11), PSNR performance attained 8.01. For BCH code (15.7) PSNR performance obtained when Eb/NO $9 \mathrm{~dB}$ is 13.90 and BCH code for (127.64) PSNR performance obtained is 23.328. Then, when using Reed Solomon code (15.11) PSNR performance obtained amounted to 7.66 and when Reed Solomon code $(31,15)$ PSNR performance obtained amounted to 16. For visualization of comparative performance of channel coding can be seen in Table 1 where the performance of the BCH code $(127,64)$ is the best of all.

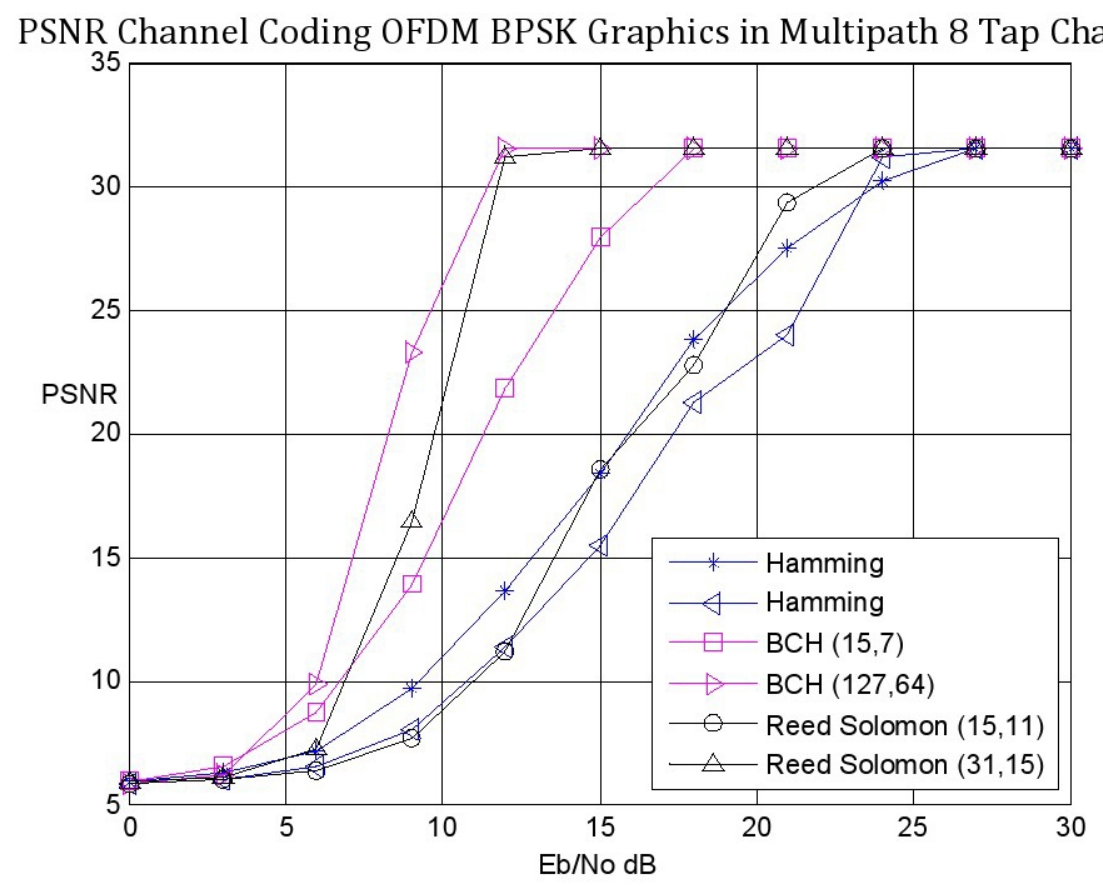

Figure 4. ComparisonofPSNR performancechannel codingOFDM system using modulation BPSK at underwater channel

Table 1.Comparisonvisualofchannel coding

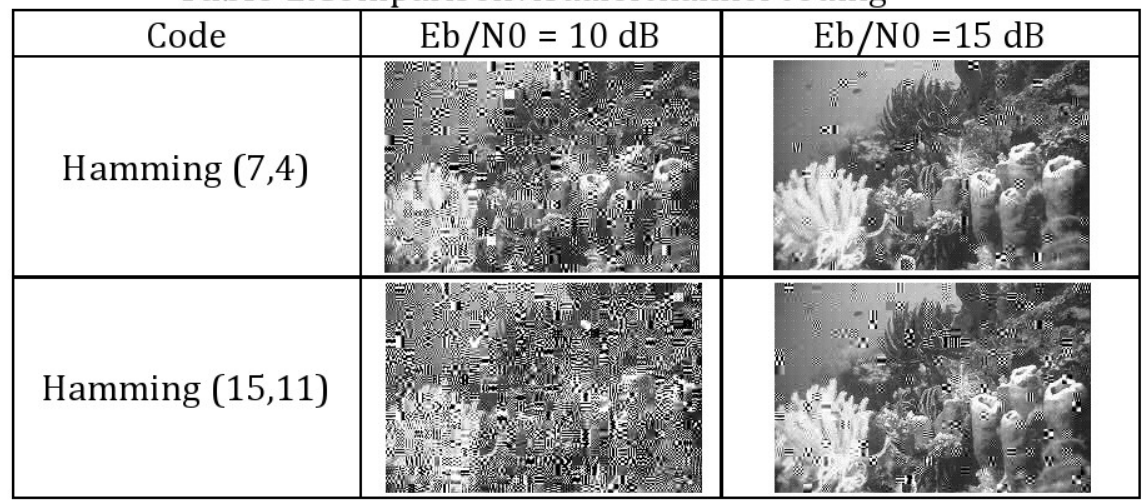


Table 1. Comparis on visual of channel coding

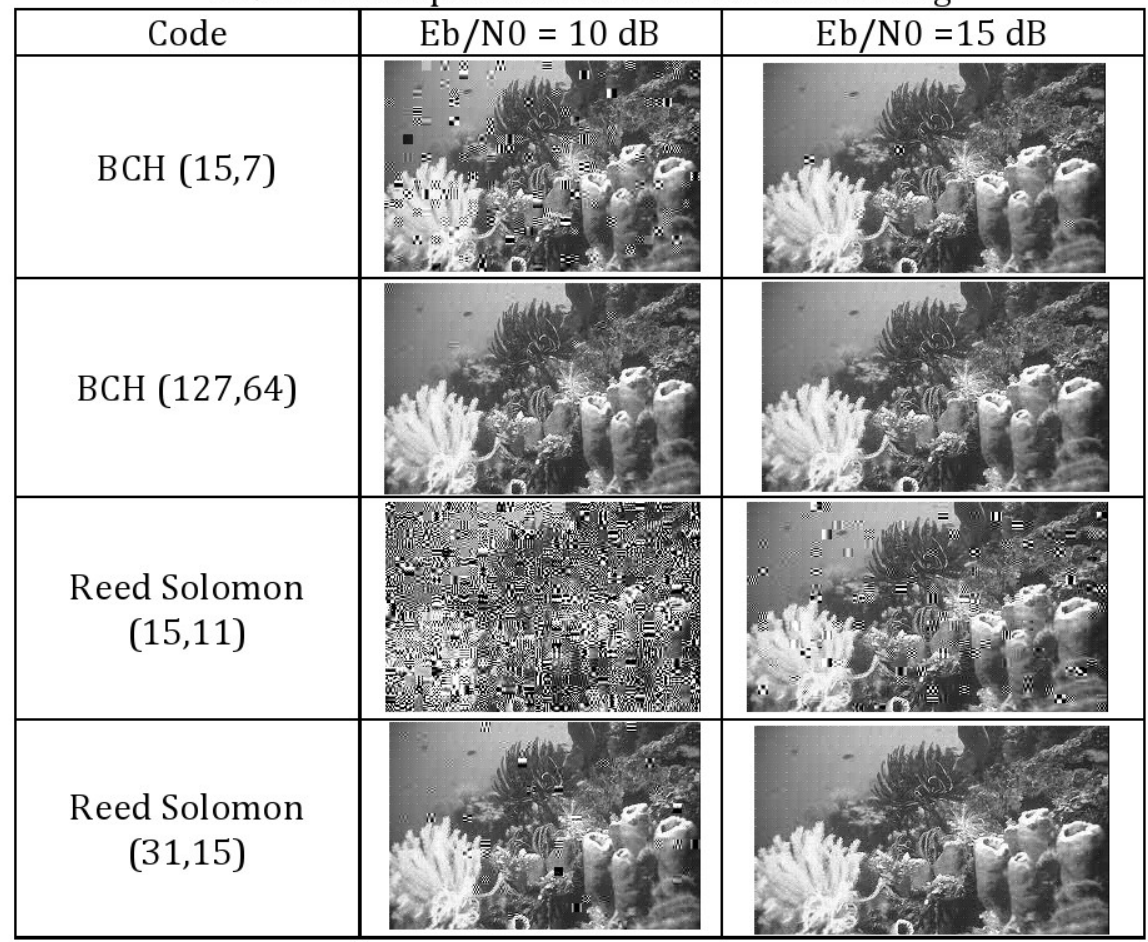

\section{DISCUSSION}

This research usesOFDM system and compares three kinds of channel codingin the underwater multipathchannel. Hamming code has decoding complexity relatively simple so that its time computation is relatively fast but the performance is not really good, inversely with $\mathrm{BCH}$ code with complicated decoding complexity so that it causes long time computation but best performance.Reed Solomon codes hasa simpler decoding complexity than $\mathrm{BCH}$ code and causes the computation time more quickly, despite that Solomon had Reed code performance is good enough. Computing time could be improved by changing the structure of the code where the code structure affects the coding rate (the larger the coding rate faster computing time) and for some codes, code structure also affects the code performance because every code structure has different error correction ability $(\mathrm{BCH}$ and $\mathrm{RS}$ code).

\section{CONCLUSION}

Based on the testing OFDM system at AWGN channel and Multipath channel, Hamming code $(7,4)$ has better performance 1 to $2 \mathrm{~dB}$ than Hamming code (15.11) for each condition of the channel (AWGN or Multipath). BCH Code $(127,64)$ has better performance $2 \mathrm{~dB}$ to $3 \mathrm{~dB}$ than BCH Code (15.7) for each channel condition (AWGN or Multipath). RS Code $(31,15)$ has better performance $3 \mathrm{~dB}$ to $6 \mathrm{~dB}$ than RS code $(15.11)$ for each condition of the channel (AWGN or Multipath). 
For all, BCH code $(127,64)$ has the best performance of all coding used started from Hamming code up to Reed Solomon code for each channel condition(AWGN orMultipath).

\section{ACKNOWLEDGMENT}

Authorswould like to say thanks a lot to the research and society dedication unit (UPPM) PENS that has given financial support so that this paper is able to be published in Emitter journal.

\section{REFERENCE}

[1] F Alkydiz, Dario Pompoli, TomasoMelodia, Underwater Acoustic Sensor Network: Research Chalenges, Elsavier, Ad Hoc Networks, pp.257-279, 2005.

[2] MandarChitre, Shiraz Shahabudeen, MilicaStojanovic, Underwater Acoustic Communications and Networking: Recent Advances and Future Challenges, Marine Technology Society Journal, Vol42, No.1, pp.103-116, 2008.

[3] Manjula.R.B, Sunilkumar S. Manvi, Issues in Underwater Acoustic Sensor Networks, International Journal of Computer and Electrical Engineering, Vol.3, No.1, pp.101-110, 2011.

[4] JordiRibas, Daniel Sura, Underwater Wireless Video Transmission for Supervisory Control and Inspection using Acoustic OFDM, December 2010.

[5] JordiRibas, Underwater Wireless Video Transmission using Acoustic OFDM, LAP LAMBERT Academic Publishing, 2009.

[6] J.H. Cui, J. Kong, M. Gerla, and S. Zhou, Challenges: Building scalable mobile underwater wireless sensor networks for aquatic applications, IEEE Network, Special Issue on Wireless Sensor Networking, pp. 12-18, 2006.

[7] Yuan Jiang, A Practical Guide to Error-Control Coding Using MATLAB, Artech House, Har/Cdr edition, 2010.

[8] Ken Cabeen, Peter Gent, Image Compression and The Discrete Cosine Transform, College of Redwoods, Report number: Math 45, 2009

[9] Yong Song Cho,MIMO-OFDM Wireless Communication with Matlab, Wiley-IEEE Press,1 edition, 2010.

[10] M.Stojanovic, OFDM For Underwater Acoustic Communications: Adaptive Synchronization and Sparse Channel Estimation, ICASSP, pp. $5288-5291,2008$. 